\title{
Ultraviolet Imaging Telescope Observations of the Magellanic Clouds
}

\author{
Joel Wm. Parker
}

Southwest Research Institute, Boulder, CO 80302, USA

Jesse K. Hill, Robert Cornett, Joan Hollis, Emily Zamkoff

Raytheon STX Corporation, NASA's GSFC, Greenbelt, MD 20771, USA

Ralph C. Bohlin

STScI, 3700 San Martin Dr., Baltimore, MD 21218, USA

Robert W. O'Connell

Astronomy Dept., Univ. of Virginia, Charlottesville, VA 22903, USA

Susan G. Neff, Andrew M. Smith, Theodore P. Stecher

Laboratory for Astronomy and Solar Physics, NASA's GSFC, Greenbelt, $M D$ 20771, USA

Morton S. Roberts

National Radio Astronomy Observatory, Charlottesville, VA 22903, USA

\begin{abstract}
.
We present an analysis of wide-field, far-ultraviolet images of the LMC and SMC obtained by the Ultraviolet Imaging Telescope. The photometric catalog of over 37,000 stars allows us to make large-scale, statistical studies of massive star formation in OB associations and in the field population. Our results show that: (1) the most probable slope for the initial mass function (IMF) of field stars is $\Gamma=-1.80$, slightly steeper than the Salpeter slope; and (2) there doesn't seem to be a single, unique IMF slope for stars in $\mathrm{OB}$ associations, with a range of values from $\Gamma=-1.0$ to -2.0 . We also analyze the stellar vs. diffuse UV flux, and the population of OB star candidates in the field.
\end{abstract}

The Ultraviolet Imaging Telescope (UIT) obtained wide-field ( $37^{\prime}$ diameter), far-ultraviolet (FUV: $1300-1800 \AA$ ) images of 19 regions in the LMC and SMC. These images provide an extensive FUV mosaic of the SMC and contain numerous regions in the LMC, covering a wide range of stellar densities and current star formation activity. A total of 47 LMC/Lucke-Hodge (1970) and 37 SMC/Hodge (1985) OB associations are completely or partially included in the observed fields. Of the $\mathrm{H}$ II regions in the DEM catalog (Davies et al. 1976), the 
UIT fields completely or partially include $102 \mathrm{DEM}$ regions in the LMC and 74 $\mathrm{DEM}$ regions in the SMC.

In this paper we focus on the catalog of 37,333 stars in the LMC (Analyses of the UIT SMC data are presented by Cornett et al. [1994, 1997], and additional analyses of specific LMC regions observed by UIT are presented by Cheng et al. [1992], J. Hill et al. (1993, 1994), R. Hill et al. [1995], and Parker et al. [1996]), with a completeness limit of $m_{\mathrm{UV}} \approx 15 \mathrm{mag}$ and a detection limit of $m_{\mathrm{UV}} \approx 17.5$. FUV magnitudes for all the stars in these images were derived from point spread function photometry. The average uncertainty in the photometry is $\sim 0.1 \mathrm{mag}$. Point-source profiles had a FWHM $\sim 3.4^{\prime \prime}$.

We divided the catalog into field stars and stars that are in DEM regions. We analyzed each of these two sets of stars independently, comparing the composite UV luminosity function of our data to UV magnitudes derived from stellar evolution and atmosphere models in order to derive the underlying stellar formation parameters. We find a most probable initial mass function (IMF) slope for the LMC field stars of $\Gamma=-1.80 \pm 0.09$, though we also find some evidence for a field star IMF slope of $\Gamma \sim-1.4$, equal to the Salpeter (1955, ApJ, 121, 161 ) slope. However, in the case of the stars in the DEM regions (the stars in all the regions were analyzed together as a single group), we find three IMF slopes of roughly equal likelihood: $\Gamma=-1.0,-1.6$, and -2.0 .

In our analysis of the DEM regions, we find that stars typically contribute $75 \%$ of the total, integrated flux in a region, the remainder being due to a diffuse UV background and/or faint stars.

Using our data in conjunction with UV models and observations to deduce the UV magnitudes of "typical" O stars and evolved later-type stars, we estimate there are many as 1100-1500 candidate O-type stars are in the DEM regions, and 1600-2300 such stars are in the general LMC field.

This paper gives an overview of our analyses and results; the full paper with finder charts, details of the data reduction, and the complete analysis is presented by Parker et al. (1998).

\section{References}

Cheng, K.-P., et al. 1992, ApJ, 395, L29

Cornett, R. H., et al. 1994, ApJ, 430, L117

Cornett, R. H., et al. 1997, AJ, 113, 1011

Davies, R. D., Elliott, K. H., Meaburn, J. 1976, MmRAS, 81, 89 (DEM)

Hill, J. K., et al. 1993, ApJ, 413, 604

Hill, J. K., et al. 1994, ApJ, 425, 122

Hill, R. S., et al. 1995, ApJ, 446, 622

Hodge, P. W. 1985, PASP, 97, 530

Lucke, P. B., Hodge, P. W. 1970, AJ, 75, 171

Parker, J. Wm., et al. 1996, ApJ, 472, L29

Parker, J. Wm., et al. 1998 AJ, 116, 180

Salpeter, E.E. 1955, ApJ, 121, 161 